Research Article

\title{
Whether Economic Freedom Is Significantly Related to Death of COVID-19
}

\author{
Ray-Ming Chen (iD \\ School of Mathematics and Statistics, Baise University, 21 Zhongshan No. 2 Road, Baise, Guangxi, China \\ Correspondence should be addressed to Ray-Ming Chen; baotaoxi@163.com
}

Received 29 October 2020; Revised 23 November 2020; Accepted 26 November 2020; Published 12 December 2020

Academic Editor: Saverio Maietta

Copyright (c) 2020 Ray-Ming Chen. This is an open access article distributed under the Creative Commons Attribution License, which permits unrestricted use, distribution, and reproduction in any medium, provided the original work is properly cited.

COVID-19 has caused a huge mayhem globally. Different economic freedom leads to different performances of a country's reaction to the pandemic. We study 164 countries and apply mathematical and statistical approaches to tackle the problem: whether economic freedom has a significant impact on the death of COVID-19. We devise a metric, some norms, and some orderings to construct an absolute reference and the actual relation via binary sequences. Then, we use the theoretical binary sequences to construct a probability distribution which linearises the strength of relation between economic freedom and death of COVID-19. Then, the actual relation from the data analysis provides an evidence to the hypothetical testing. Our analysis and model show that there is no significant relation between economic freedom and death of COVID-19.

\section{Introduction}

Due to COVID-19 pandemic, there are many fatalities across the world. Many countries are baffled by whether to open the market or impose lockdown [1-3]. It creates a huge chaos in either economic or social stability $[4,5]$. This motivates us to study the relation between economic freedom and the death rate or tolls of COVID-19. We locate 164 countries from some datasets $[6,7]$-because some of the countries lack statistics of either the economic freedom or the death information regarding COVID-19. Then, we use a series of mathematical and statistical approaches to reach a conclusion. For the mathematical part, we define a new concept of metric $d$ which could measure the difference between the scoring structures-this is hardly the case if one adopts the usual Euclidean metric. For reference purpose, one fixes the referential structure $\vec{e}$ (or scoring system) first. Then, one could compute the distances between all the (sampled) multivalued data $N$ points $\left\{\vec{v}_{i}: 1 \leq i \leq N\right\}$ and $I$, i.e., $\left\{d\left(\vec{e}, \vec{v}_{i}\right): 1 \leq i \leq N\right\}$. Based on these distances, we could then create an ordering for $\left\{\vec{v}_{i}: 1 \leq i \leq N\right\}$ with respect to the referential structure $\vec{e}$.

\section{Modelling}

2.1. Notations and Symbols. For a vector $\vec{w}$, we use $|\vec{w}|$ to denote its length; for any set $H$, we use $|H|$ to denote its size (cardinality). Moreover, we use $\vec{w}(j)$ to denote the $j$-th element in $\vec{w}$. Let $\vec{b}$ denote a binary vector, i.e., each element in $\vec{b}$ is either 0 or 1 . Let $\mathbb{B}_{k}$ denote the set of all the binary vectors with total length $k$. Let $\mathbb{C}=\left\{C_{1}, C_{2}, \ldots, C_{m}\right\} \quad$ be $a$ set of countries. Let $A_{e f}=\left\{A_{1}, A_{2}, \ldots, A_{n}\right\}$ be a set of attributes of economic freedom (regarded as independent variables). Let $B_{j}$ be a set of result (regarded as dependent variables). Each time we fix one $B_{j}$ to study the relation between the attributes and $B_{j}$. In this article, we restrict our attribute values to be numerical numbers. The theoretical table is shown in Table 1, and for the actual forms, one could refer to Sections 3.2.1 and 3.2.2. 
TABLE 1: Independent-dependent analysis.

\begin{tabular}{lllllllll}
\hline Countries & $A_{1}$ & $A_{2}$ & $\ldots$ & $A_{p}$ & $B_{1}$ & $B_{2}$ & $\ldots$ & $B_{q}$ \\
\hline$C_{1}$ & $a_{11}$ & $a_{12}$ & $\ldots$ & $a_{1 p}$ & $b_{11}$ & $b_{12}$ & $\ldots$ & $B_{1 q}$ \\
$C_{2}$ & $a_{21}$ & $a_{22}$ & $\ldots$ & $a_{2 p}$ & $b_{21}$ & $b_{22}$ & $\ldots$ & $b_{2 q}$ \\
$\ldots$ & & & & & & & & \\
$C_{m}$ & $a_{m 1}$ & $a_{m 2}$ & $\ldots$ & $a_{m p}$ & $b_{m 1}$ & $b_{m 2}$ & $\ldots$ & $b_{m q}$ \\
\hline
\end{tabular}

We use the notations $\overrightarrow{C_{i}}=\left(a_{i 1}, a_{i 2}, \ldots, a_{i m}\right) ; \overrightarrow{A_{i}}=\left(a_{1 i}\right.$, $\left.a_{2 i}, \ldots, a_{m i}\right)$; and $\overrightarrow{B_{i}}=\left(b_{1 i}, b_{2 i}, \ldots, b_{m i}\right)$.

\subsection{Binary Subvectors and Norm}

Definition 1 (subvectors). Suppose $\vec{b}$ is a binary vector. We use Sub $(\vec{b})$ to denote all its truncated subvectors consisting of only 1 .

Example 1. Suppose $\vec{b}=(1,0,1,0,0,1,1,1,0,1,1,0,1,0,1$ $, 1,0,0,0,1,1,0,1,0)$. Then, $\operatorname{Sub}(\vec{b})=\{(1),(1),(1,1,1)$, $(1,1),(1),(1,1),(1,1),(1)\}$.

We simply abbreviate it as $\operatorname{Sub}(\vec{b})=1^{1} 1^{1} 1^{3} 1^{2} 1^{1} 1^{2} 1^{2} 1^{1}$. Indeed $\operatorname{Sub}(\vec{b})$ reveals the structure of an independentdependent variable relation.

Definition 2 (binary norm). For any binary vector $\vec{b}=\left(b_{1}, b_{2}, \ldots, \underline{b_{k}}\right)$ with $\operatorname{Sub}(\vec{b})=1^{n_{1}} 1^{n_{2}}, \ldots, 1^{n_{t}}$, define a binary norm $\|\vec{b}\|=\left(2^{0}+2^{1}+\cdots+2^{n_{1}-1}\right)+\left(2^{0}+2^{1}+\right.$ $\left.\cdots+2^{n_{2}-1}\right)+\cdots+\left(2^{0}+2^{1}+\cdots+2^{n_{t}-1}\right)$.

One could, according to real situations, adopt other numbers (for example, replace 2 with other numbers) or other forms other than the one provided here.

Claim 1. $\|\vec{b}\|=\left(2^{n_{1}}+2^{n_{2}}+\cdots+2^{n_{t}}\right)-t$.

Proof. It follows immediately from the definition.

Definition 3 (linear ordering on $\mathbb{B}_{k}$ ) $\cdot \vec{b}_{1} \geq \vec{b}_{2}$ if and only if $\left\|\vec{b}_{1}\right\| \geq\left\|\vec{b}_{2}\right\|$, for all $\vec{b}_{1}, \vec{b}_{2} \in \mathbb{B}_{k}$.

Example 2. If $\vec{b}_{1}=(1,0,1,1,1,0,0,1,1,0,1,0,1), \vec{b}_{2}=$ $(1,0,0,0,0,1,1,0,0,1,1,1,1)$, then $\left\|\vec{b}_{1}\right\|=\left(2^{0} \cdot 1\right)+\left(2^{0}\right.$. $\left.1+2^{1} \cdot 1+2^{2} \cdot 1\right)+\left(2^{0} \cdot 1+2^{1} \cdot 1\right)+\left(2^{0} \cdot 1\right)+\left(2^{0} \cdot 1\right)=13$ and $\left\|\vec{b}_{2}\right\|=\left(\begin{array}{ll}2^{0} & \cdot 1\end{array}\right)+\left(2^{0} \cdot 1+2^{1} \cdot 1\right)+\left(2^{0} \cdot 1+2^{1} \cdot 1+\right.$ $\left.2^{2} \cdot 1+2^{3} \cdot 1\right)=19$. Thus, $\vec{b}_{2} \leq \vec{b}_{1}$.

Remark 1. A binary norm indeed serves an important technique in revealing the relation between dependent and independent variables. Given two pairs $\left(x_{1}, y_{1}\right)$ and $\left(x_{2}, y_{2}\right)$ of numerical data with $x_{1} \neq x_{2}$, if $\left(x_{2}-x_{1}\right) \cdot\left(y_{2}-y_{1}\right)>0$ (i.e., they act proportionally), we associate it with a value 1 to indicate such relation and 0 , otherwise (i.e., they act inversely). Such mechanism gives a way to look into the fundamental relation between $X$ and $Y$ variables. This kind of analysis is in particular useful when the precision of the data is questionable or when the actual numbers are unknown or more suitable to be interpreted via ranks.

Example 3 (sign vector). Suppose $D=((2,4),(3,2)$, $(5,8),(7,9),(4,2),(3,8))$, a set of ordered vectors. Then, we could associate $D$ with a sign vector $\vec{v}=(0,1,1,1,0)$ via Remark 1.

Definition 4 (relational vector). Suppose that $\vec{b}$ is a sign vector; we associate it with a relational vector $\operatorname{Rel}(\vec{b})$ whose $i$-th element is assigned 1 iff $\vec{b}(i)=\vec{b}(i+1)$ and 0 , otherwise.

Example 4. Let us continue with Example 3. We could compute its relational vector $\operatorname{Rel}(\vec{v})=(0,1,1,0)$, and thus $\operatorname{Sub}(\operatorname{Rel}(\vec{v}))=1^{2}$ and $\|\operatorname{Sub}(\operatorname{Rel}(\vec{v}))\|=3$. The higher the value of the norm is, the closer the relation between the dependent and independent variables is.

$\underset{\text { Definition }}{\rightarrow}$ (equivalence relation $\sim$ ). For all $\vec{b}_{1}, \vec{b}_{2} \in \mathbb{B}_{k}$, $\vec{b}_{1} \sim \vec{b}_{2}$ iff $\left\|\vec{b}_{1}\right\|=\left\|\vec{b}_{2}\right\|$.

Let $\mathbb{B}_{k}^{\#}=\left\{\|\vec{b}\|: \vec{b} \in \mathbb{B}_{k}\right\}$. One observes that $\sim$ partitions $\mathbb{B}_{k}$. If $p \in \mathbb{B}_{k}^{\#}$, we use $[p]$ to denote the equivalence class whose elements' norms are all $p$.

2.3. Probability Distribution. Suppose $\mathbb{B}_{k}=\{0,1\}^{k}$ is the sampling population. Define a statistic $\mathrm{BN}$ on $\mathbb{B}_{k}$ by its binary norm. The range for $\mathrm{BN}$ is $\mathbb{B}_{k}^{\#}$. Define a counting $\rho: \mathbb{B}_{k}^{\#} \longrightarrow \mathbb{N}$ by $\rho(x)=\left|\left\{\vec{b} \in \mathbb{B}_{k}:\|\vec{b}\|=x\right\}\right|$. Now, we could define the probability distribution for $\mathrm{BN}$ by prob: $\mathbb{B}_{k}^{\#} \longrightarrow[0,1]$ by

$$
\operatorname{prob}(u)=\frac{\rho(u)}{\sum_{h \in \operatorname{Ran}} \rho(h)} .
$$

One observes that

$$
\operatorname{prob}(u)=\frac{|[u]|}{\sum_{h \in \operatorname{Ran}}|[h]|} .
$$

This probability distribution reveals the relation between the independent variables and the dependent variables. This would serve our theoretical distribution for our statistical testing $\mathrm{H}_{0}$ : the economic freedom and the death of COVID19 has no significant relation, i.e., the economic freedom has no great impact on the death of COVID-19. For a concrete construction of such probability distribution, one could refer to Section 6.1.

2.4. Metric. A metric or a distance function is a non-negative function $d$ on $X \times X$ satisfying identity, symmetry, and triangle properties. In this article, it suffices to define a metric on a closed interval of real number. Fix $I=[a, b] \subseteq \mathbb{R}$, where $a, b \in \mathbb{R}$ and $a<b$. Let $\vec{v}$ be a finite vector whose first element is $a$, last element is $b$, and all the other elements are incrementally increased and lie between $a$ and $b$. Let 
$\operatorname{FIN}[a, b]$ be the set of all such vectors. Let $\vec{v}=\left(a, v_{2}, \ldots, v_{m-1}, b\right), \vec{w}=\left(a, w_{2}, w_{3}, \ldots, w_{n-1}, b\right) \in$ Fin $[a, b]$ be arbitrary. Let $\vec{v} \sqcap \vec{w}$ denote the vector $q=\left(a, q_{1}, q_{2}, \ldots, q_{h-1}, b\right)$ whose elements are the projections from $\vec{v}$ and $\vec{w}$. One observes that $\operatorname{FIN}[a, b]$ is closed under $\sqcap$.

Definition 6 (atomic norm)

$\|\vec{v}\|_{E}=$

$$
\sqrt{\left(v_{2}-a\right)^{2}+\left(v_{3}-v_{2}\right)^{2}+\left(v_{4}-v_{3}\right)^{2}+\cdots\left(v_{m-2}-v_{m-1}\right)^{2}+\left(b-v_{m-1}\right)^{2}} .
$$

Definition 7 (metric). Define $d$ : $\operatorname{FIN}[a, b] \times \operatorname{FIN}[a$, b] $\longrightarrow \mathbb{R}^{+}$by

$$
d(\vec{v}, \vec{w})=\frac{\|\vec{v}\|_{E}+\|\vec{w}\|_{E}}{2}-\|\vec{v} \sqcap \vec{w}\|_{E}
$$

Example 5. Suppose the closed interval $I=[0,20]$ and $\vec{v}=$ $(0,2,4,8,19,20)$ and $\vec{w}=(0,1,4,6,12,14,15,20)$. Then, $\vec{v} \sqcap \vec{w}=(0,1,2,4,6,8,12,14,15,19,20)$. Hence, the norm $\|\vec{v}\|_{E}=\sqrt{2^{2}+2^{2}+4^{2}+11^{2}+1^{2}}=\sqrt{146} ;$ the norm $\vec{w}=$ $\sqrt{1^{2}+3^{2}+2^{2}+6^{2}+2^{2}+1^{2}+5^{2}}=\sqrt{80} ;$ and $\|\vec{v} \sqcap \vec{w}\|_{E}=$ $\sqrt{52}$. Thus, $d(\vec{v}, t \vec{w})=\sqrt{146}+\sqrt{80} / 2-\sqrt{52}=3.30$.

Claim 2. $d$ is a metric on $\operatorname{FIN}[a, b]$.

Proof. This can be shown by the definitions and some techniques.

This metric will be used in Section 3.3. This metric basically measures the differences between the structures of the attributes in the scoring system. The more similar the structures are, the lower the distances are. Unlike the static Euclidean distance, this metric takes the interval structures into consideration.

2.5. Procedures. Let us summarise the whole procedure of our modelling for the sake of data analysis. Let $\vec{e}=(100,100,100,100, \ldots, 100,100)$. Let Death $\left(C_{i}\right)$ denote the death rate (or tolls, depending on the context) for the country $i$.

(1) Define a metric $d$ on a real interval, in particular the transformed interval, an interval for attribute values which lie between 0 and $100, I=[0,1200] \subseteq \mathbb{R}$ for the range of attribute values of economic freedom, and calculate $\left\{d\left(\widehat{I}, \vec{C}_{i}\right): 1 \leq i \leq m\right\}$ (one could refer to Section 3.3).

(2) Rank $\mathbb{C}$ via the sorted distances with a rank function $\gamma_{100}: \mathbb{C} \longrightarrow\{1,2, \ldots, m\}$ in which $\gamma_{I}\left(C_{i}\right) \geq \gamma_{I}\left(C_{j}\right)$ iff $d\left(\vec{I}, \vec{C}_{i}\right) \geq d\left(\vec{I}, \vec{C}_{i}\right)$.

(3) Rank $\mathbb{C}$ via the sorted distance with a rank function $\gamma_{88}$ in which $\gamma_{88}\left(C_{i}\right) \geq \gamma_{88}\left(C_{j}\right)$ iff Death $\left(C_{i}\right)$ $\geq \operatorname{Death}\left(C_{j}\right)$.

(4) Form the vector $\vec{v}=\left(\gamma_{100}{ }^{\circ} \gamma_{88}^{-1}(1)\right)_{l=1}^{m}$.
(5) Convert $\vec{v}$ into a sign vector sg $(\vec{v}):=(\chi(\vec{v}(2)-$ $\vec{v}(1)), \chi(\vec{v}(3)-\vec{v}(2)), \ldots, \chi(\vec{v}(m)-\vec{v}(m-1)$ ) ), where $\chi(a)=1$ if $a>0$ and $\chi(a)=0$ if $a<0$.

(6) Construct the probability distribution for the quotient space $\mathbb{B}_{k} / \sim$.

(7) Perform statistical testing by locating the position of $\vec{v}$ and significant level for the batch of country.

(8) Apply the Monte Carlo approach on the sampled batches of countries repeatedly.

(9) With the threshold probability 0.5 , based on binary distribution for the whole spectrum of statistical testing, perform the overall statistical testing.

(10) Draw a conclusion for the relation between $\gamma_{100}$ and $\gamma_{88}$.

\section{Data Analysis}

Following the procedures in Section 2.5, we start to collect, analyse, and produce a report via data analysis. Since the data are huge and hard to handle by the one-off approach, we resort to the sampling technique and reach a conclusion via statistical testing.

3.1. Sampling. The raw data consist of 164 countries (we use 1 to 164 to name the countries) up to 2020 , June $27^{\text {th }}$. Since the size is too huge, we apply the Monte Carlo approach to sample the 164 countries. We do 20 times (or 20 batches: S1 to S20) sampling with 25 countries over the 164 countries per sampling. The sampled batches are listed in Tables 2 and 3.

\subsection{Sampled Data}

3.2.1. Economic Freedom. Corresponding to the form listed in Table 1, we associate $\mathbb{C}$ with $\mathrm{S} 1$ and define $A_{e f}=\{A 1, A 2, \ldots, A 12\}$, where $A 1 \equiv$ Property Rights, $A 2 \equiv$ Judicial Effectiveness, $A 3 \equiv$ Government Integrity, $A 4 \equiv$ Tax Burden, A5 $\equiv$ Government Spending, $A 6 \equiv$ Fiscal Health, $A 7 \equiv$ Business Freedom, $A 8 \equiv$ Labor Freedom, $A 9 \equiv$ Monetary Freedom, $A 10 \equiv$ Trade Freedom, $A 11 \equiv$ Investment Freedom, and $A 12 \equiv$ Financial Freedom. The attribute values are based on a 100 -point scoring system [6]. Due to the limitation of space, we list only the first sampling (or S1) regarding its attributes of economic freedom in Table 4 . We omit the other 19 similar tables of this form. $A_{\text {ef }}$ serves as the set of our independent variables. 
TABle 2: 20 sampled batches-S1 to S10.

\begin{tabular}{lcccccccccc}
\hline Order & S1 & S2 & S3 & S4 & S5 & S6 & S7 & S8 & S9 & S10 \\
\hline 1 & 68 & 25 & 69 & 10 & 27 & 65 & 72 & 56 & 64 & 163 \\
2 & 141 & 127 & 93 & 98 & 55 & 85 & 75 & 151 & 150 & 19 \\
3 & 21 & 95 & 5 & 56 & 14 & 115 & 159 & 112 & 65 & 10 \\
4 & 129 & 41 & 30 & 45 & 7 & 35 & 130 & 136 & 9 & 117 \\
5 & 127 & 17 & 151 & 74 & 119 & 57 & 128 & 139 & 69 & 52 \\
6 & 99 & 151 & 33 & 89 & 20 & 160 & 147 & 100 & 27 & 18 \\
7 & 89 & 14 & 156 & 26 & 124 & 95 & 19 & 27 & 113 & 88 \\
8 & 2 & 43 & 132 & 73 & 35 & 118 & 122 & 83 & 53 & 65 \\
9 & 128 & 76 & 44 & 84 & 153 & 29 & 129 & 16 & 84 & 105 \\
10 & 108 & 28 & 48 & 70 & 150 & 15 & 9 & 47 & 110 & 150 \\
11 & 112 & 62 & 126 & 67 & 151 & 71 & 118 & 134 & 156 & 26 \\
12 & 92 & 131 & 6 & 121 & 90 & 86 & 91 & 87 & 89 & 161 \\
13 & 98 & 10 & 46 & 69 & 80 & 89 & 39 & 107 & 52 & 82 \\
14 & 18 & 119 & 84 & 50 & 37 & 50 & 71 & 52 & 21 & 27 \\
15 & 148 & 128 & 39 & 97 & 84 & 13 & 81 & 111 & 132 & 57 \\
16 & 124 & 121 & 34 & 3 & 45 & 56 & 15 & 48 & 15 & 29 \\
17 & 1 & 18 & 95 & 92 & 75 & 91 & 158 & 101 & 66 & 20 \\
18 & 32 & 16 & 98 & 18 & 113 & 41 & 125 & 46 & 127 & 116 \\
19 & 76 & 92 & 26 & 87 & 59 & 7 & 144 & 113 & 56 & 38 \\
20 & 14 & 42 & 85 & 142 & 85 & 32 & 46 & 70 & 97 & 41 \\
21 & 41 & 60 & 1 & 37 & 48 & 61 & 21 & 76 & 140 & 92 \\
22 & 137 & 103 & 144 & 137 & 38 & 75 & 86 & 84 & 102 & 128 \\
23 & 85 & 120 & 53 & 2 & 132 & 124 & 132 & 119 & 31 & 7 \\
24 & 78 & 163 & 117 & 122 & 111 & 122 & 99 & 74 & 163 & 22 \\
25 & 52 & 118 & 62 & 77 & 66 & 19 & 35 & 123 & 88 & 103 \\
\hline
\end{tabular}

TABle 3: 20 sampled batches-S11 to S20.

\begin{tabular}{lcccccccccc}
\hline Order & S11 & S12 & S13 & S14 & S15 & S16 & S17 & S18 & S19 & S20 \\
\hline 1 & 76 & 91 & 83 & 59 & 64 & 119 & 129 & 154 & 15 & 37 \\
2 & 23 & 124 & 53 & 63 & 46 & 123 & 8 & 82 & 30 & 55 \\
3 & 29 & 151 & 126 & 24 & 143 & 137 & 6 & 35 & 43 & 119 \\
4 & 12 & 126 & 129 & 55 & 53 & 108 & 122 & 4 & 148 & 163 \\
5 & 73 & 65 & 9 & 143 & 86 & 54 & 152 & 40 & 76 & 155 \\
6 & 157 & 86 & 18 & 100 & 59 & 76 & 7 & 69 & 133 & 18 \\
7 & 49 & 54 & 102 & 50 & 40 & 6 & 120 & 19 & 8 & 6 \\
8 & 68 & 119 & 50 & 85 & 52 & 156 & 93 & 89 & 146 & 99 \\
9 & 15 & 87 & 104 & 74 & 32 & 152 & 70 & 146 & 145 & 122 \\
10 & 48 & 8 & 74 & 40 & 68 & 111 & 27 & 143 & 57 & 162 \\
11 & 104 & 11 & 87 & 115 & 36 & 101 & 25 & 134 & 26 & 25 \\
12 & 99 & 67 & 125 & 79 & 135 & 85 & 136 & 70 & 101 & 156 \\
13 & 142 & 59 & 158 & 52 & 156 & 3 & 66 & 38 & 131 & 147 \\
14 & 136 & 139 & 20 & 159 & 39 & 37 & 110 & 61 & 31 & 10 \\
15 & 41 & 43 & 57 & 69 & 96 & 30 & 67 & 20 & 20 & 134 \\
16 & 115 & 148 & 151 & 108 & 50 & 66 & 50 & 142 & 6 & 79 \\
17 & 151 & 56 & 54 & 49 & 27 & 51 & 53 & 54 & 63 & 44 \\
18 & 77 & 15 & 27 & 36 & 47 & 162 & 26 & 98 & 25 & 157 \\
19 & 126 & 16 & 90 & 136 & 154 & 124 & 132 & 56 & 70 & 4 \\
20 & 108 & 82 & 93 & 87 & 132 & 95 & 89 & 151 & 85 & 20 \\
21 & 95 & 39 & 161 & 98 & 138 & 138 & 44 & 111 & 71 & 67 \\
22 & 60 & 37 & 44 & 21 & 62 & 120 & 76 & 113 & 118 & 153 \\
23 & 36 & 6 & 16 & 54 & 120 & 69 & 141 & 108 & 84 & 97 \\
24 & 1 & 42 & 86 & 109 & 12 & 134 & 84 & 7 & 41 & 148 \\
25 & 74 & 10 & 103 & 119 & 157 & 136 & 73 & 52 & 58 & 136 \\
\hline & & & & & & & & & &
\end{tabular}

3.2.2. COVID-19. Now we start to introduce the dependent variables. Indeed we tackle an individual dependent variable each time. Since the data are huge, we only extract the data [7] for sampling one (or S1) as shown in Table 5.
Corresponding to the form listed in Table 1, we associate $\mathbb{C}$ with S1 and define $B_{1} \equiv$ Total Confirmed COVID-19 Cases, $B_{2} \equiv$ Death Toll of COVID-19, $B_{3} \equiv$ Total Recovered COVID-19 Cases, and $B_{4} \equiv$ Population of the Countries. Due to the limitation of space, we list only the first sampling (or S1) regarding its dependent variables. We omit the other 19 similar tables of this form. Moreover, in the later analysis, we only take and fix $B_{2}$ as our dependent variable. If the readers are interested in other dependent variables (or $B_{1}$, $B_{3}$, or other mixed forms), they could simply follow the same approach provided in this article.

3.3. Metric. Since we have defined an interval metric in Section 2.4, we could apply it over here. Here we measure the distance between every sampled data and the fixed reference vector $\vec{e}=(100,200, \ldots, 1100,1200)$. We construct the distances for the 164 countries based on economic freedom (for example, the data of sample one could be referred from Table 4) in Tables 6 and 7. Since all the data are presented in the form of 100-point score for the attribute values in Table 4, we need to transform the values in the table to the interval $I=[0,1200]$. For example, the reference vector $\vec{e}$ (we still use $\vec{e}$ to represent to newly transformed vector) will be $\vec{e}=(0,100,200,300, \ldots, 1100,1200)$. Each country $C$ sampled in S1 will be transformed into $\vec{C}$, for example, $\overrightarrow{\vec{C}}_{11} \equiv \overrightarrow{68}=(0,64.8,145.7,247.5,379.9, \ldots, 1170,1200)$ are the converted data for the first country sampled in the first sampling or country 68. The economic freedom vector for each sampled country is converted by the same way. The converted data are not tabulated. Then, we apply $d$ in Section 2.4 on the converted data and repeat the whole processes for other samplings. The complete results regarding the distance for the 20 sampled countries are presented in Tables 6 and 7. The $(i, j)$ cell in the tables means the value $d\left(\vec{e}, \vec{C}_{i j}\right)$, where $\underline{C}_{i j}$ denotes the $i$-th country sampled in $j$-th sampling and $\underline{\vec{C}}_{i j}$ denotes the converted data for $\underline{C}_{i j}$.

\section{Absolute Reference}

By the derived distances presented in Tables 6 and 7, we could construct absolute references. The absolute references would server as the benchmarks for other internal structures. Let us use $\mathbb{C}_{s}$ to denote the set of sampled countries in $s$-th sampling. Let $\overrightarrow{\underline{C}}_{s i}, \underline{\underline{C}}_{s j} \in \mathbb{C}_{s}$ be arbitrary.

Definition 8 (ordering of the sampled countries). $\underline{C}_{i s} \geq \underline{C}_{j s}$ iff $d\left(\vec{e}, \underline{\vec{C}}_{i s}\right) \geq d\left(\vec{e}, \underline{\vec{C}}_{j s}\right)$.

Based on this ordering, we could generate the absolute references (Tables 8 and 9). Let us take S1 for example: $C_{68}>C_{112}>C_{92}>\cdots>C_{41}>C_{85}>C_{14}$. From these absolute references (or ordering for the samplings), we could view the structure (or interval) difference between the ideal scoring (or $\vec{e}$ ) and real scoring results. Indeed, an absolute reference is a reference acting like ordering without specific scales. Such reference is useful when the precise values are unknown or when the precision of the data is questionable. In 


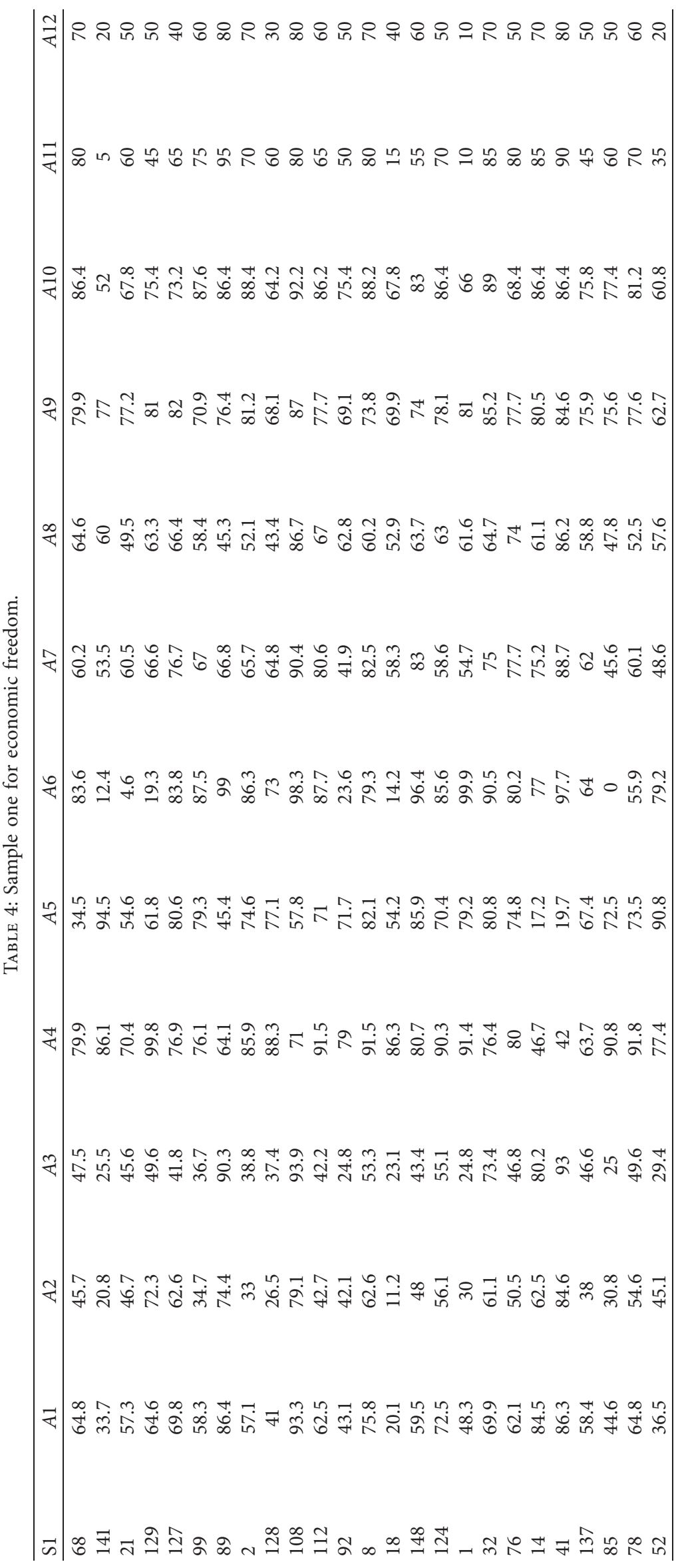


TABle 5: Sample one for COVID-19.

\begin{tabular}{lccccc}
\hline S1 & $\mathbb{C}$ & $B_{1}$ & $B_{2}$ & $B_{3}$ & $B_{4}$ \\
\hline 68 & Hungary & 4127 & 578 & 2663 & 9660521 \\
141 & Sudan & 9257 & 572 & 4014 & 43828543 \\
21 & Brazil & 1280054 & 56109 & 697526 & 212541690 \\
129 & Senegal & 6354 & 98 & 4193 & 16734279 \\
127 & Sao Tome and & 712 & 13 & 219 & 219087 \\
99 & Principe & & & & \\
89 & Mexico & 208392 & 25779 & 120562 & 128914507 \\
2 & Luxembourg & 4173 & 110 & 3968 & 625810 \\
128 & Albania & 2269 & 51 & 1298 & 2877821 \\
108 & Saudi Arabia & 174577 & 1474 & 120471 & 34805142 \\
112 & New Zealand & 1520 & 22 & 1484 & 5002100 \\
92 & North Macedonia & 5758 & 268 & 2206 & 2083375 \\
98 & Malawi & 1005 & 13 & 260 & 19119281 \\
18 & Mauritius & 341 & 10 & 326 & 1271749 \\
148 & Bolivia & 28503 & 913 & 7338 & 11670618 \\
124 & Thailand & 3162 & 58 & 3040 & 69798329 \\
1 & Romania & 25697 & 1579 & 18181 & 19238321 \\
32 & Afghanistan & 30451 & 683 & 10306 & 38910996 \\
76 & Chile & 263360 & 5068 & 223431 & 19114153 \\
14 & Jamaica & 686 & 10 & 539 & 2961033 \\
41 & Belgium & 61106 & 9731 & 16918 & 11589102 \\
137 & Denmark & 12675 & 604 & 11508 & 5792000 \\
85 & South Africa & 124590 & 2340 & 64111 & 59297807 \\
78 & Lebanon & 1697 & 33 & 1144 & 6825627 \\
52 & Jordan & 1104 & 9 & 830 & 10201800 \\
\hline & Ethiopia & 5425 & 89 & 1688 & 114903773 \\
\hline & & & & &
\end{tabular}

TABLE 6: Distance function for 20 samplings-S1 to S10.

\begin{tabular}{lccccccccc}
\hline S1 & S2 & S3 & S4 & S5 & S6 & S7 & S8 & S9 & S10 \\
\hline 61.0 & 80.1 & 82.2 & 74.2 & 78.3 & 73.2 & 53.9 & 59.2 & 66.8 & 72.2 \\
76.2 & 76.8 & 75.4 & 72.4 & 73.6 & 84.0 & 72.8 & 83.4 & 80.4 & 71.3 \\
70.7 & 72.4 & 71.1 & 59.2 & 85.1 & 68.6 & 66.5 & 62.3 & 73.2 & 74.2 \\
80.8 & 82.1 & 71.4 & 82.3 & 64.9 & 77.2 & 74.3 & 85.7 & 70.2 & 65.0 \\
76.8 & 71.8 & 83.4 & 78.5 & 72.8 & 67.1 & 76.9 & 78.9 & 82.2 & 80.7 \\
75.0 & 83.4 & 69.7 & 65.5 & 84.4 & 49.2 & 67.7 & 77.4 & 78.3 & 75.8 \\
65.5 & 85.1 & 57.3 & 71.3 & 71.1 & 72.4 & 71.3 & 78.3 & 74.4 & 61.8 \\
80.4 & 70.4 & 74.8 & 68.7 & 77.2 & 65.0 & 67.8 & 67.3 & 57.5 & 73.2 \\
76.9 & 69.5 & 81.5 & 74.8 & 73.1 & 71.8 & 80.8 & 73.1 & 74.8 & 68.1 \\
73.3 & 61.3 & 60.1 & 71.4 & 80.4 & 83.2 & 70.2 & 72.3 & 72.7 & 80.4 \\
62.3 & 73.8 & 71.9 & 76.5 & 83.4 & 64.2 & 65.0 & 77.6 & 57.3 & 71.3 \\
63.0 & 74.9 & 49.9 & 77.8 & 71.9 & 80.1 & 82.6 & 62.7 & 65.5 & 82.3 \\
72.4 & 74.2 & 74.0 & 82.2 & 78.4 & 65.5 & 66.0 & 45.7 & 80.7 & 72.5 \\
75.8 & 72.8 & 74.8 & 79.9 & 54.1 & 79.9 & 64.2 & 80.7 & 70.7 & 78.3 \\
68.8 & 76.9 & 66.0 & 65.1 & 74.8 & 70.4 & 74.4 & 69.2 & 74.8 & 67.1 \\
71.1 & 77.8 & 77.3 & 71.4 & 82.3 & 59.2 & 83.2 & 60.1 & 83.2 & 71.8 \\
71.3 & 75.8 & 72.4 & 63.0 & 72.8 & 82.6 & 71.6 & 75.4 & 33.6 & 84.4 \\
74.3 & 73.1 & 72.4 & 75.8 & 74.4 & 82.1 & 68.8 & 74.0 & 76.8 & 74.5 \\
69.5 & 63.0 & 71.3 & 62.7 & 82.6 & 64.9 & 59.4 & 74.4 & 59.2 & 74.0 \\
85.1 & 77.7 & 84.0 & 59.7 & 84.0 & 74.3 & 74.0 & 71.4 & 65.1 & 82.1 \\
82.1 & 66.0 & 71.3 & 54.1 & 60.1 & 72.0 & 70.7 & 69.5 & 64.2 & 63.0 \\
67.7 & 82.7 & 59.4 & 67.7 & 74.0 & 72.8 & 80.1 & 74.8 & 82.3 & 76.9 \\
84.0 & 73.3 & 57.5 & 80.4 & 74.8 & 71.1 & 74.8 & 72.8 & 60.9 & 64.9 \\
67.7 & 72.2 & 65.0 & 67.8 & 69.2 & 67.8 & 75.0 & 78.5 & 72.2 & 68.2 \\
80.7 & 65.0 & 73.8 & 78.6 & 33.6 & 71.3 & 77.2 & 72.3 & 61.8 & 82.7 \\
\hline & & & & & & & & &
\end{tabular}

TABle 7: Distance function for 20 samplings-S11 to S20.

\begin{tabular}{llllllllll}
\hline S11 & S12 & S13 & S14 & S15 & S16 & S17 & S18 & S19 & S20 \\
\hline 69.5 & 82.6 & 67.3 & 82.6 & 66.8 & 72.8 & 80.8 & 81.1 & 83.2 & 54.1 \\
82.3 & 71.1 & 57.5 & 83.2 & 74.0 & 72.3 & 71.6 & 72.5 & 71.4 & 73.6 \\
71.8 & 83.4 & 71.9 & 74.4 & 52.9 & 67.7 & 49.9 & 77.2 & 70.4 & 72.8 \\
68.7 & 71.9 & 80.8 & 73.6 & 57.5 & 73.3 & 67.8 & 90.3 & 68.8 & 72.2 \\
68.7 & 73.2 & 70.2 & 52.9 & 80.1 & 71.6 & 80.6 & 49.1 & 69.5 & 58.9 \\
62.9 & 80.1 & 75.8 & 77.4 & 82.6 & 69.5 & 64.9 & 82.2 & 32.3 & 75.8 \\
59.6 & 71.6 & 82.3 & 79.9 & 49.1 & 49.9 & 73.3 & 71.3 & 71.6 & 49.9 \\
61.0 & 72.8 & 79.9 & 84.0 & 80.7 & 57.3 & 75.4 & 65.5 & 73.5 & 75.0 \\
83.2 & 62.7 & 78.8 & 78.5 & 74.3 & 80.6 & 71.4 & 73.5 & 80.1 & 67.8 \\
60.1 & 71.6 & 78.5 & 49.1 & 61.0 & 69.2 & 78.3 & 52.9 & 67.1 & 82.0 \\
78.8 & 84.0 & 62.7 & 68.6 & 80.7 & 75.4 & 80.1 & 77.6 & 71.3 & 80.1 \\
75.0 & 76.5 & 68.8 & 83.9 & 74.5 & 84.0 & 85.7 & 71.4 & 75.4 & 57.3 \\
59.7 & 82.6 & 71.6 & 80.7 & 57.3 & 71.4 & 33.6 & 74.0 & 74.9 & 67.7 \\
85.7 & 78.9 & 84.4 & 66.5 & 66.0 & 54.1 & 72.7 & 72.0 & 60.9 & 74.2 \\
82.1 & 70.4 & 67.1 & 82.2 & 72.7 & 71.4 & 76.5 & 84.4 & 84.4 & 77.6 \\
68.6 & 68.8 & 83.4 & 73.3 & 79.9 & 33.6 & 79.9 & 59.7 & 49.9 & 83.9 \\
83.4 & 59.2 & 71.6 & 59.6 & 78.3 & 74.1 & 57.5 & 71.6 & 83.2 & 81.5 \\
78.6 & 83.2 & 78.3 & 80.7 & 72.3 & 82.0 & 71.3 & 72.4 & 80.1 & 62.9 \\
71.9 & 73.1 & 71.9 & 85.7 & 81.1 & 71.1 & 74.8 & 59.2 & 71.4 & 90.3 \\
73.3 & 72.5 & 75.4 & 62.7 & 74.8 & 72.4 & 65.5 & 83.4 & 84.0 & 84.4 \\
72.4 & 66.0 & 82.3 & 72.4 & 78.2 & 78.2 & 81.5 & 69.2 & 64.2 & 76.5 \\
66.0 & 54.1 & 81.5 & 70.7 & 73.8 & 73.3 & 69.5 & 74.4 & 65.0 & 73.1 \\
80.7 & 49.9 & 73.1 & 71.6 & 73.3 & 82.2 & 76.2 & 73.3 & 74.8 & 65.1 \\
71.3 & 77.7 & 80.1 & 84.7 & 68.7 & 77.6 & 74.8 & 64.9 & 82.1 & 68.8 \\
78.5 & 74.2 & 82.7 & 72.8 & 62.9 & 85.7 & 68.7 & 80.7 & 82.9 & 85.7 \\
\hline & & & & & & & & &
\end{tabular}

TABLE 8: Absolute references for 20 samplings-S1 to S10.

\begin{tabular}{lccccccccc}
\hline S1 & S2 & S3 & S4 & S5 & S6 & S7 & S8 & S9 & S10 \\
\hline 68 & 28 & 6 & 37 & 66 & 160 & 72 & 107 & 66 & 88 \\
112 & 92 & 156 & 56 & 37 & 56 & 144 & 56 & 156 & 92 \\
92 & 118 & 53 & 142 & 48 & 71 & 71 & 48 & 53 & 7 \\
89 & 60 & 144 & 87 & 7 & 7 & 118 & 112 & 56 & 117 \\
78 & 76 & 48 & 92 & 111 & 118 & 39 & 87 & 31 & 57 \\
137 & 43 & 117 & 97 & 124 & 89 & 159 & 83 & 88 & 105 \\
148 & 17 & 39 & 89 & 90 & 57 & 147 & 111 & 140 & 22 \\
76 & 163 & 33 & 137 & 75 & 122 & 122 & 76 & 97 & 26 \\
21 & 95 & 5 & 122 & 119 & 115 & 125 & 70 & 89 & 19 \\
124 & 119 & 1 & 73 & 153 & 13 & 9 & 123 & 64 & 29 \\
1 & 16 & 26 & 26 & 55 & 124 & 21 & 47 & 9 & 163 \\
98 & 120 & 30 & 70 & 38 & 19 & 19 & 119 & 21 & 82 \\
108 & 62 & 126 & 3 & 113 & 29 & 158 & 16 & 163 & 65 \\
32 & 10 & 98 & 98 & 132 & 61 & 75 & 46 & 110 & 38 \\
99 & 131 & 95 & 10 & 84 & 95 & 46 & 113 & 65 & 10 \\
18 & 18 & 62 & 84 & 35 & 75 & 130 & 84 & 113 & 116 \\
141 & 127 & 46 & 18 & 27 & 65 & 81 & 101 & 132 & 18 \\
127 & 128 & 132 & 67 & 80 & 32 & 132 & 100 & 84 & 128 \\
128 & 42 & 84 & 121 & 150 & 35 & 99 & 134 & 127 & 27 \\
2 & 121 & 93 & 74 & 45 & 50 & 128 & 27 & 27 & 150 \\
52 & 25 & 34 & 77 & 59 & 86 & 35 & 74 & 150 & 52 \\
129 & 41 & 44 & 50 & 151 & 41 & 86 & 139 & 52 & 41 \\
41 & 103 & 69 & 2 & 85 & 91 & 129 & 52 & 69 & 161 \\
85 & 151 & 151 & 69 & 20 & 15 & 91 & 151 & 102 & 103 \\
14 & 14 & 85 & 45 & 14 & 85 & 15 & 136 & 15 & 20 \\
\hline & & & & & & & & &
\end{tabular}


TABLE 9: Absolute references for 20 samplings-S11 to S20.

\begin{tabular}{lccccccccc}
\hline S11 & S12 & S13 & S14 & S15 & S16 & S17 & S18 & S19 & S20 \\
\hline 49 & 6 & 53 & 40 & 40 & 66 & 66 & 40 & 133 & 6 \\
142 & 37 & 87 & 143 & 143 & 6 & 6 & 143 & 6 & 37 \\
48 & 56 & 57 & 49 & 156 & 37 & 53 & 56 & 31 & 156 \\
68 & 87 & 83 & 87 & 53 & 156 & 7 & 142 & 71 & 155 \\
157 & 39 & 125 & 159 & 68 & 137 & 89 & 7 & 118 & 157 \\
60 & 148 & 9 & 115 & 157 & 111 & 122 & 89 & 57 & 97 \\
115 & 43 & 158 & 21 & 39 & 76 & 73 & 111 & 148 & 147 \\
73 & 124 & 54 & 54 & 64 & 124 & 76 & 19 & 76 & 122 \\
12 & 8 & 126 & 98 & 12 & 30 & 26 & 70 & 43 & 148 \\
76 & 54 & 90 & 119 & 47 & 3 & 70 & 54 & 26 & 163 \\
1 & 126 & 16 & 108 & 96 & 54 & 8 & 61 & 70 & 119 \\
29 & 82 & 93 & 55 & 120 & 123 & 110 & 98 & 30 & 153 \\
126 & 119 & 18 & 24 & 62 & 95 & 120 & 82 & 8 & 55 \\
95 & 16 & 27 & 100 & 46 & 119 & 132 & 108 & 146 & 10 \\
108 & 65 & 74 & 74 & 32 & 120 & 84 & 146 & 84 & 99 \\
99 & 10 & 104 & 50 & 135 & 108 & 93 & 38 & 131 & 18 \\
74 & 67 & 50 & 52 & 132 & 51 & 141 & 113 & 101 & 67 \\
77 & 42 & 86 & 36 & 138 & 101 & 67 & 35 & 145 & 134 \\
104 & 139 & 129 & 69 & 27 & 134 & 27 & 134 & 25 & 25 \\
36 & 86 & 44 & 59 & 50 & 138 & 50 & 52 & 41 & 44 \\
41 & 91 & 102 & 63 & 86 & 152 & 25 & 154 & 58 & 162 \\
23 & 59 & 161 & 79 & 52 & 162 & 152 & 69 & 63 & 79 \\
15 & 15 & 103 & 85 & 36 & 69 & 129 & 151 & 15 & 20 \\
151 & 151 & 151 & 109 & 154 & 85 & 44 & 20 & 85 & 136 \\
136 & 11 & 20 & 136 & 59 & 136 & 136 & 4 & 20 & 4 \\
\hline & & & & & & & & &
\end{tabular}

TABLE 10: Ordering for each batch based on death toll of COVID19-S1 to S10.

\begin{tabular}{lccccccccc}
\hline S1 & S2 & S3 & S4 & S5 & S6 & S7 & S8 & S9 & S10 \\
\hline 78 & 43 & 53 & 67 & 90 & 15 & 15 & 83 & 53 & 117 \\
98 & 131 & 117 & 98 & 20 & 35 & 35 & 101 & 15 & 20 \\
76 & 25 & 126 & 26 & 35 & 118 & 118 & 151 & 102 & 22 \\
127 & 151 & 151 & 69 & 151 & 57 & 91 & 76 & 69 & 26 \\
92 & 17 & 69 & 142 & 150 & 91 & 39 & 16 & 140 & 92 \\
108 & 76 & 98 & 92 & 84 & 160 & 147 & 134 & 64 & 150 \\
85 & 127 & 26 & 84 & 48 & 85 & 159 & 84 & 127 & 57 \\
2 & 92 & 39 & 87 & 85 & 56 & 132 & 48 & 150 & 163 \\
148 & 118 & 62 & 56 & 132 & 50 & 129 & 87 & 163 & 161 \\
52 & 16 & 84 & 2 & 38 & 65 & 19 & 56 & 84 & 82 \\
129 & 163 & 48 & 50 & 59 & 7 & 9 & 52 & 56 & 10 \\
89 & 62 & 85 & 10 & 7 & 89 & 130 & 136 & 132 & 88 \\
112 & 42 & 30 & 37 & 37 & 95 & 81 & 123 & 110 & 38 \\
141 & 10 & 132 & 89 & 80 & 19 & 128 & 47 & 31 & 52 \\
68 & 95 & 95 & 97 & 113 & 13 & 122 & 113 & 88 & 65 \\
41 & 60 & 93 & 74 & 66 & 41 & 144 & 112 & 52 & 7 \\
1 & 103 & 156 & 3 & 111 & 61 & 46 & 74 & 65 & 19 \\
18 & 28 & 6 & 18 & 124 & 122 & 71 & 100 & 89 & 103 \\
128 & 41 & 1 & 77 & 45 & 124 & 125 & 111 & 97 & 116 \\
124 & 18 & 44 & 121 & 153 & 71 & 72 & 46 & 9 & 41 \\
137 & 120 & 5 & 122 & 119 & 115 & 99 & 107 & 113 & 18 \\
32 & 121 & 144 & 73 & 14 & 32 & 75 & 119 & 156 & 128 \\
14 & 128 & 46 & 137 & 55 & 29 & 21 & 70 & 66 & 29 \\
99 & 119 & 34 & 45 & 75 & 75 & 158 & 139 & 21 & 105 \\
21 & 14 & 33 & 70 & 27 & 86 & 86 & 27 & 27 & 27 \\
\hline & & & & & & & & &
\end{tabular}

TABLE 11: Ordering for each batch based on death toll of COVID19-S11 to S20.

\begin{tabular}{lccccccccc}
\hline S11 & S12 & S13 & S14 & S15 & S16 & S17 & S18 & S19 & S20 \\
\hline 49 & 43 & 83 & 49 & 53 & 101 & 53 & 20 & 43 & 162 \\
15 & 126 & 53 & 69 & 12 & 162 & 25 & 35 & 101 & 25 \\
126 & 15 & 90 & 98 & 96 & 51 & 67 & 151 & 131 & 20 \\
104 & 67 & 20 & 36 & 64 & 76 & 26 & 4 & 20 & 67 \\
12 & 151 & 126 & 63 & 36 & 69 & 76 & 69 & 25 & 4 \\
151 & 16 & 104 & 108 & 39 & 134 & 84 & 142 & 15 & 134 \\
76 & 91 & 151 & 159 & 135 & 108 & 152 & 98 & 145 & 163 \\
142 & 39 & 102 & 85 & 62 & 85 & 132 & 134 & 76 & 147 \\
36 & 87 & 57 & 87 & 132 & 30 & 110 & 108 & 26 & 148 \\
108 & 56 & 16 & 24 & 50 & 152 & 50 & 56 & 118 & 10 \\
48 & 82 & 87 & 50 & 52 & 37 & 129 & 82 & 57 & 37 \\
136 & 42 & 161 & 109 & 59 & 123 & 7 & 146 & 63 & 136 \\
95 & 148 & 50 & 52 & 47 & 136 & 136 & 38 & 133 & 97 \\
60 & 10 & 129 & 59 & 138 & 95 & 89 & 52 & 84 & 79 \\
23 & 65 & 93 & 136 & 156 & 138 & 93 & 7 & 85 & 156 \\
74 & 59 & 9 & 79 & 40 & 156 & 6 & 89 & 30 & 6 \\
68 & 37 & 103 & 74 & 68 & 54 & 66 & 19 & 146 & 44 \\
41 & 54 & 74 & 54 & 120 & 6 & 141 & 113 & 148 & 18 \\
1 & 6 & 54 & 40 & 46 & 66 & 8 & 54 & 31 & 155 \\
77 & 8 & 44 & 100 & 32 & 111 & 44 & 40 & 6 & 122 \\
73 & 124 & 18 & 115 & 143 & 3 & 120 & 111 & 41 & 153 \\
115 & 11 & 125 & 143 & 157 & 120 & 122 & 61 & 8 & 119 \\
29 & 119 & 158 & 119 & 86 & 124 & 73 & 143 & 71 & 99 \\
99 & 139 & 27 & 55 & 27 & 137 & 70 & 70 & 58 & 55 \\
157 & 86 & 86 & 21 & 154 & 119 & 27 & 154 & 70 & 157 \\
\hline & & & & & & & & &
\end{tabular}

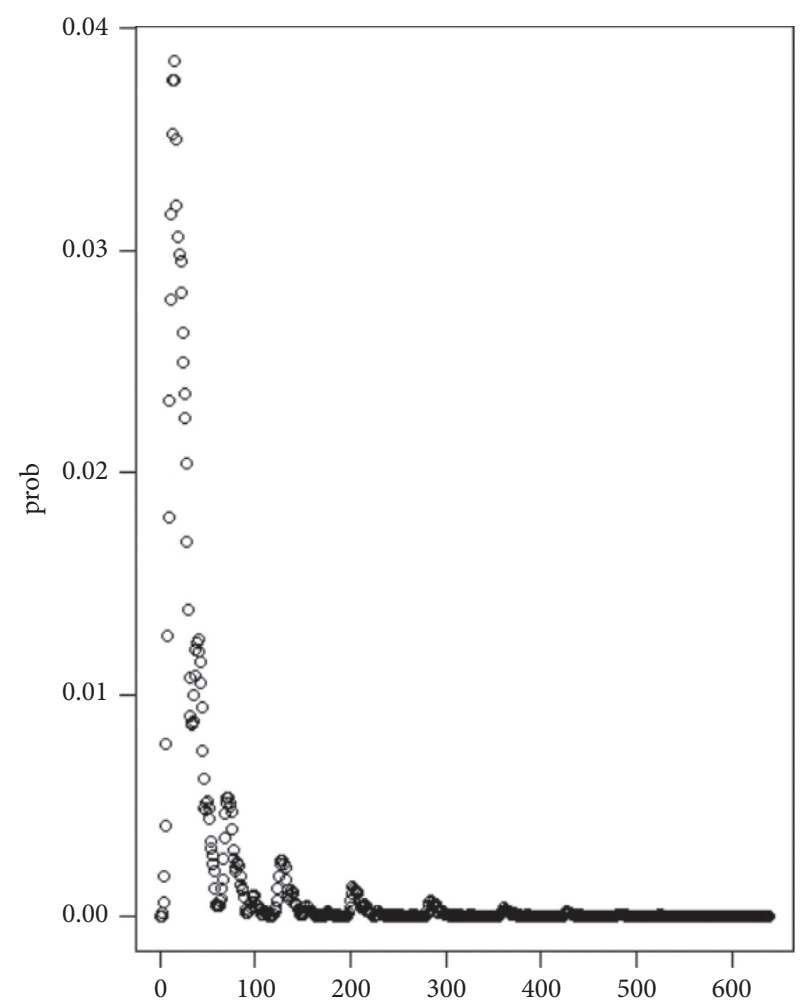

FIGURE 1: Theoretical distribution of binary norm. 
this article, we use relative distances between a country's economic freedom and others to create such ordering.

\section{Ordering for COVID-19 Fatalities}

Based on Table 5 and other omitted tables, we start to construct the ordering (or ranking) based on the fatalities of COVID-19.

Definition 9 (ordering on fatalities). $\quad \underline{C}_{i s} \geq \underline{C}_{j s}$ iff Death $\left(\underline{C}_{i s}\right) \geq \operatorname{Death}\left(\underline{C}_{j s}\right)$, where Death $\left(\underline{C}_{i s}\right)$ is the death toll for $i$-th country sampled in $s$-th sampling.

Based on this ordering, we have the results presented in Tables 10 and 11. Let us take the cells in S1 for example: $C_{78}>C_{98}>C_{76}>\cdots>C_{14}>C_{99}>C_{21}$.

\section{Norm and Probability}

In this experiment, we only consider $N=23$ and construct its distribution accordingly. Hence, the domain is $\{0,1\}^{23}$ and the range lies between 0 and $2^{23}-1=8388607$ (indeed some of the values' probability is 0 ). This section generalises Example 3. The higher the value is, the higher the impact of independent variables on dependent variable is.

6.1. Probability Distribution. We have already constructed the theoretical setting of probability distribution for our testing in Section 2.3. Based on that framework and the data given, we could create the theoretical probability distribution prob in Figure 1.

The (one-tailed) critical values for 5 and 10 percentages are 138 and 78 (via numerical computation), respectively; that is, if the sampled value is larger than the critical values, we should reject $H_{o}$ : there is no significant relation between the economic freedom and death of COVID-19.

6.2. Real Results. In comparison with the absolute reference, we could generate the binary sign vectors for the real data from each sampling $S_{j}$ (or simply $j$ ) in Table 12-for the formula and explanation of sign vectors, one could refer to Section 2.2. However, in these 0 and 1 representations, it separates the proportional and inversely proportional relation between the economic freedom and death of COVID19. To take all the factors into consideration, one further analyses the alternative behaviour of 0 and 1 . If there are too many alternations between 0 and 1 , it would indicate that there is a less relation between those two. On the other hand, if the alternative times are few, then it leads to the longer length of subvector consisting of pure 1 . The alternative results are shown in Table 13.

Based on Table 13 and definitions in Section 2.2, we could compute the binary norm for each sampling batch $S_{j}$ (or $J$ ) as shown in Table 14 .
TABLE 12: Sign vectors.

\begin{tabular}{cccccccccccccccccccc}
\hline 1 & 2 & 3 & 4 & 5 & 6 & 7 & 8 & 9 & 10 & 11 & 12 & 13 & 14 & 15 & 16 & 17 & 18 & 19 & 20 \\
\hline 1 & 1 & 1 & 0 & 1 & 0 & 0 & 1 & 1 & 1 & 1 & 1 & 0 & 1 & 1 & 1 & 1 & 0 & 1 & 0 \\
0 & 1 & 1 & 0 & 0 & 0 & 0 & 1 & 0 & 0 & 0 & 1 & 1 & 0 & 1 & 0 & 0 & 1 & 0 & 1 \\
1 & 1 & 1 & 1 & 1 & 1 & 1 & 0 & 0 & 1 & 1 & 0 & 1 & 1 & 0 & 0 & 0 & 1 & 1 & 0 \\
0 & 0 & 0 & 0 & 0 & 1 & 0 & 1 & 0 & 0 & 0 & 1 & 0 & 1 & 1 & 1 & 0 & 0 & 0 & 1 \\
1 & 0 & 0 & 1 & 0 & 0 & 1 & 1 & 1 & 1 & 1 & 0 & 1 & 0 & 0 & 0 & 1 & 0 & 1 & 0 \\
1 & 1 & 0 & 1 & 0 & 1 & 0 & 0 & 1 & 0 & 0 & 1 & 1 & 0 & 1 & 0 & 1 & 1 & 0 & 0 \\
0 & 0 & 0 & 0 & 1 & 0 & 1 & 0 & 1 & 1 & 0 & 0 & 0 & 1 & 0 & 1 & 0 & 1 & 0 & 0 \\
0 & 1 & 1 & 0 & 0 & 1 & 1 & 1 & 0 & 1 & 1 & 0 & 0 & 0 & 1 & 0 & 0 & 0 & 1 & 1 \\
1 & 1 & 1 & 1 & 0 & 0 & 0 & 0 & 1 & 0 & 0 & 0 & 1 & 1 & 1 & 1 & 1 & 0 & 0 & 1 \\
1 & 0 & 0 & 0 & 1 & 0 & 0 & 1 & 0 & 1 & 0 & 1 & 0 & 1 & 1 & 0 & 1 & 1 & 1 & 0 \\
0 & 1 & 1 & 0 & 0 & 1 & 1 & 1 & 1 & 0 & 1 & 1 & 1 & 1 & 1 & 1 & 0 & 1 & 1 & 1 \\
0 & 1 & 0 & 0 & 0 & 1 & 1 & 0 & 0 & 1 & 0 & 0 & 0 & 0 & 0 & 1 & 1 & 1 & 0 & 0 \\
1 & 0 & 1 & 1 & 1 & 0 & 1 & 1 & 0 & 1 & 0 & 1 & 1 & 1 & 1 & 0 & 0 & 1 & 1 & 1 \\
0 & 0 & 0 & 0 & 0 & 0 & 0 & 1 & 1 & 0 & 1 & 0 & 0 & 1 & 0 & 1 & 1 & 0 & 1 & 0 \\
1 & 0 & 1 & 1 & 0 & 1 & 0 & 0 & 1 & 0 & 0 & 1 & 0 & 0 & 0 & 0 & 0 & 1 & 0 & 0 \\
0 & 1 & 0 & 0 & 1 & 0 & 1 & 1 & 0 & 1 & 0 & 0 & 1 & 0 & 1 & 1 & 0 & 1 & 1 & 1 \\
1 & 0 & 0 & 1 & 1 & 0 & 0 & 0 & 0 & 1 & 1 & 1 & 0 & 0 & 1 & 0 & 1 & 1 & 0 & 0 \\
1 & 1 & 1 & 1 & 1 & 1 & 1 & 0 & 0 & 0 & 0 & 0 & 0 & 0 & 1 & 0 & 0 & 0 & 0 & 0 \\
0 & 0 & 1 & 0 & 0 & 0 & 0 & 1 & 1 & 1 & 1 & 1 & 1 & 1 & 1 & 1 & 1 & 0 & 0 & 1 \\
0 & 0 & 0 & 0 & 0 & 1 & 1 & 0 & 1 & 0 & 0 & 0 & 0 & 0 & 0 & 1 & 0 & 1 & 1 & 1 \\
1 & 1 & 0 & 1 & 1 & 1 & 0 & 1 & 0 & 1 & 0 & 1 & 0 & 0 & 1 & 1 & 0 & 1 & 0 & 0 \\
1 & 0 & 1 & 0 & 0 & 0 & 0 & 0 & 0 & 0 & 1 & 0 & 1 & 1 & 1 & 0 & 1 & 0 & 0 & 1 \\
0 & 0 & 1 & 1 & 0 & 1 & 1 & 1 & 1 & 0 & 1 & 1 & 1 & 1 & 0 & 0 & 1 & 1 & 1 & 0 \\
0 & 1 & 0 & 0 & 1 & 1 & 1 & 0 & 1 & 1 & 0 & 1 & 1 & 0 & 1 & 1 & 1 & 1 & 0 & 0 \\
\hline
\end{tabular}

TABLE 13: Relational vectors.

\begin{tabular}{cccccccccccccccccccc}
\hline 1 & 2 & 3 & 4 & 5 & 6 & 7 & 8 & 9 & 10 & 11 & 12 & 13 & 14 & 15 & 16 & 17 & 18 & 19 & 20 \\
\hline 0 & 1 & 1 & 1 & 0 & 1 & 1 & 1 & 0 & 0 & 0 & 1 & 0 & 0 & 1 & 0 & 0 & 0 & 0 & 0 \\
0 & 1 & 1 & 0 & 0 & 0 & 0 & 0 & 1 & 0 & 0 & 0 & 1 & 0 & 0 & 1 & 1 & 1 & 0 & 0 \\
0 & 0 & 0 & 0 & 0 & 1 & 0 & 0 & 1 & 0 & 0 & 0 & 0 & 1 & 0 & 0 & 1 & 0 & 0 & 0 \\
0 & 1 & 1 & 0 & 1 & 0 & 0 & 1 & 0 & 0 & 0 & 0 & 0 & 0 & 0 & 0 & 0 & 1 & 0 & 0 \\
1 & 0 & 1 & 1 & 1 & 0 & 0 & 0 & 1 & 0 & 0 & 0 & 1 & 1 & 0 & 1 & 1 & 0 & 0 & 1 \\
0 & 0 & 1 & 0 & 0 & 0 & 0 & 1 & 1 & 0 & 1 & 0 & 0 & 0 & 0 & 0 & 0 & 1 & 1 & 1 \\
1 & 0 & 0 & 1 & 0 & 0 & 1 & 0 & 0 & 1 & 0 & 1 & 1 & 0 & 0 & 0 & 1 & 0 & 0 & 0 \\
0 & 1 & 1 & 0 & 1 & 0 & 0 & 0 & 0 & 0 & 0 & 1 & 0 & 0 & 1 & 0 & 0 & 1 & 0 & 1 \\
1 & 0 & 0 & 0 & 0 & 1 & 1 & 0 & 0 & 0 & 1 & 0 & 0 & 1 & 1 & 0 & 1 & 0 & 0 & 0 \\
0 & 0 & 0 & 1 & 0 & 0 & 0 & 1 & 0 & 0 & 0 & 1 & 0 & 1 & 1 & 0 & 0 & 1 & 1 & 0 \\
1 & 1 & 0 & 1 & 1 & 1 & 1 & 0 & 0 & 0 & 0 & 0 & 0 & 0 & 0 & 1 & 0 & 1 & 0 & 0 \\
0 & 0 & 0 & 0 & 0 & 0 & 1 & 0 & 1 & 1 & 1 & 0 & 0 & 0 & 0 & 0 & 0 & 1 & 0 & 0 \\
0 & 1 & 0 & 0 & 0 & 1 & 0 & 1 & 0 & 0 & 0 & 0 & 0 & 1 & 0 & 0 & 0 & 0 & 1 & 0 \\
0 & 1 & 0 & 0 & 1 & 0 & 1 & 0 & 1 & 1 & 0 & 0 & 1 & 0 & 1 & 0 & 0 & 0 & 0 & 1 \\
0 & 0 & 0 & 0 & 0 & 0 & 0 & 0 & 0 & 0 & 1 & 0 & 0 & 1 & 0 & 0 & 1 & 1 & 0 & 0 \\
0 & 0 & 1 & 0 & 1 & 1 & 0 & 0 & 1 & 1 & 0 & 0 & 0 & 1 & 1 & 0 & 0 & 1 & 0 & 0 \\
1 & 0 & 0 & 1 & 1 & 0 & 0 & 1 & 1 & 0 & 0 & 0 & 1 & 1 & 1 & 1 & 0 & 0 & 1 & 1 \\
0 & 0 & 1 & 0 & 0 & 0 & 0 & 0 & 0 & 0 & 0 & 0 & 0 & 0 & 1 & 0 & 0 & 1 & 1 & 0 \\
1 & 1 & 0 & 1 & 1 & 0 & 0 & 0 & 1 & 0 & 0 & 0 & 0 & 0 & 0 & 1 & 0 & 0 & 0 & 1 \\
0 & 0 & 1 & 0 & 0 & 1 & 0 & 0 & 0 & 0 & 1 & 0 & 1 & 1 & 0 & 1 & 1 & 1 & 0 & 0 \\
1 & 0 & 0 & 0 & 0 & 0 & 1 & 0 & 1 & 0 & 0 & 0 & 0 & 0 & 1 & 0 & 0 & 0 & 1 & 0 \\
0 & 1 & 1 & 0 & 1 & 0 & 0 & 0 & 0 & 1 & 1 & 0 & 1 & 1 & 0 & 1 & 1 & 0 & 0 & 0 \\
1 & 0 & 0 & 0 & 0 & 1 & 1 & 0 & 1 & 0 & 0 & 1 & 1 & 0 & 0 & 0 & 1 & 1 & 0 & 1 \\
\hline
\end{tabular}

TABle 14: Binary norm for the 20 samplings: S1 to S20.

\begin{tabular}{cccccccccccccccccccc}
\hline 1 & 2 & 3 & 4 & 5 & 6 & 7 & 8 & 9 & 10 & 11 & 12 & 13 & 14 & 15 & 16 & 17 & 18 & 19 & 20 \\
\hline 8 & 11 & 15 & 8 & 11 & 8 & 9 & 6 & 14 & 5 & 6 & 6 & 9 & 15 & 17 & 8 & 11 & 17 & 7 & 8 \\
\hline
\end{tabular} 


\section{Conclusion and Future Work}

The contribution of death in COVID-19 is very complicated. We use economic freedom to capture a potential factor in such contribution. To verify the truth of great impact from economic freedom, we devise a metric, two norms, absolute ordering, binary ordering, and probability distribution for the statistical testing population. Based on our research, we find out that the economic freedom has no significant relation to the death of COVID-19. This might provide some reference for the decision makers of the countries. In the future research, one could further study the relation between economic freedom and other ratios related to COVID-19. One could also use other nonparametric approaches to enrich the statistical testing. There is another related paper on the same topic [8]. In that paper, the authors use two-step estimators: negative binomial regression and nonlinear least squares, and find out there is a close relation between economic freedom and fatalities of COVID-19. In essence, their approach focuses more on statistical techniques, while ours focuses more on mathematical approaches. For the future researcher, he could compare or combine these methods to yield a comprehensive or generalised theory that could accommodate and single out the factors that cause the discrepancies.

\section{Data Availability}

The data supporting the findings of this study are included within the article.

\section{Conflicts of Interest}

The author declares that there are no conflicts of interest.

\section{Acknowledgments}

This study was supported by the Humanities and Social Science Research Planning Fund Project under the Ministry of Education of China (grant no. 20XJAGAT001).

\section{References}

[1] S. R. Baker, N. Bloom, S. J. Davis, and S. J. Terry, COVIDInduced Economic Uncertainty, National Bureau of Economic Research, Cambridge, MA, USA, NBER Working Paper No. 26983, 2020.

[2] A. Ichino, G. Calzolari, A. Mattozzi, A. Rustichini, G. Zanella, and M. Arnelli, Transtion Steps to Stop COVID-19 without Killing the World Economy, VOX CEPR Policy Portal, London, UK, 2020.

[3] R. Meninno and G. Wolff, "As coronavirus spreads, can the EU afford to close its borders?" in Economics in the Time of COVID-19, CEPR Press, London, UK, 2020.

[4] United Nations, A UN Framework for the Immediate SocioEconomic Response to COVID-19, United Nations, New York, NY, USA, 2020.

[5] United Nations, Shared Responsibility, Global Solidarity: Responding to the Socio-Economic Impacts of COVID-19, United Nations, New York, NY, USA, 2020.
[6] The Heritage Foundation, Index of Economic Freedom, The Heritage Foundation, Washington, DC, USA, 2020, https:// www.heritage.org/index/download.

[7] https://www.worldometers.info/coronavirus/, 2020.

[8] K. Szulczyk and M. Cheema, "The impact of economic freedom on the COVID-19 fatality rate," 2020, https://www.researchgate.net/ publication/343850047_The_impact_of_economic_freedom_on_ the_COVID-19_fatality_rate. 